\title{
Essential Roles of Her2/erbB2 in Cardiac Development and Function
}

\author{
Alejandra Negro, Bhawanjit K. Brar, and Kuo-Fen Lee \\ Clayton Foundation Laboratories for Peptide Biology, The Salk Institute, \\ La Jolla, California 92037-1002
}

\begin{abstract}
The tyrosine kinase receptor erbB2, also known in humans as Her2, is a member of the epidermal growth factor receptor (EGFR or erbB1) family, which also includes erbB3 and erbB4. The erbBs were discovered in an avian erythroblastosis tumor virus and exhibited similarities to human EGFR (Yarden and Sliwkowski, 2001). Her2/erbB2 is highly expressed in many cancer types. Its overexpression is correlated with a poor prognosis for breast and ovarian cancer patients. ErbB receptors bind to a family of growth factors, termed neuregulins/heregulin (NRG/HRG), which comprise NRG-1, -2, -3, and -4 and include multiple isoforms. ErbB2/Her2 is an orphan receptor that does not bind ligand alone but heterodimerizes with the other erbB receptors for NRG signaling. ErbB2 is expressed in multiple neuronal and non-neuronal tissues in embryos and adult animals, including the heart. Genetic data demonstrated that erbB2 is required for normal embryonic development of neural crest-derived cranial sensory neurons. ErbB2/Her2-null mutant embryos of a trabeculation defect die before embryonic day (E) 11. To study its role at later stages of development, we generated a transgenic mouse line that specifically expresses the rat erbB2 cDNA in the heart under the control of the cardiac-specific $\alpha$-myosin heavy chain promoter. When crossed into the null background, the expression of the rat erbB2 cDNA rescued the cardiac phenotype in the erbB2-null mutant mice that survive until birth but display an absence of Schwann cells and a severe loss of both motor and spinal sensory neurons. To study the role of erbB2 in the adult heart, we generated conditional mutant mice carrying a cardiac-restricted deletion of erbB2. These erbB2 conditional mutants exhibited multiple independent parameters of dilated cardiomyopathy, including chamber dilation, wall thinning, and decreased contractility. Interestingly, treatment of breast cancers overexpressing erbB2 with Herceptin (Trastuzumab), a humanized monoclonal antibody specific to the extracellular domain of erbB2, results in some patients developing cardiac dysfunction. The adverse effect is increased significantly in those patients who also receive the chemotherapeutical agent anthracycline. We found that erbB2-deficient cardiac myocytes are more susceptible to anthracyclineinduced cytotoxicity. These results suggest that erbB2 signaling in the heart is essential for the prevention of dilated cardiomyopathy. These lines of mice provide models with which to elucidate the molecular and cellular mechanisms by which erbB2 signaling regulates cardiac functions. These mice also will provide important information for devising strategies to mitigate the cardiotoxic effects of Herceptin treatment, allowing for the potential expanded use of this drug to treat all cancers overexpressing erbB2.
\end{abstract}




\section{Introduction}

Cardiovascular diseases continue to be a major health risk and are the leading cause of death in both men and women (Michels et al., 2003). Dilated cardiomyopathy (DCM) is a life-threatening heart disease characterized by cardiac enlargement and decreased contractility. Gene mutations affecting cardiac myocyte contractility (e.g., sarcomeric cytoskeletal proteins) often result in the development of DCM (Chein, 1999). In the last decade, it was discovered that the neuregulin (NRG)/erbB signaling network plays a role in the development and function of the heart, including the formation of trabeculae (Gassmann et al., 1995; Lee et al., 1995; Meyer and Birchmeier, 1995), angiogenesis (Russell et al., 1999; Izumi et al., 2002), formation of heart valves (Camenisch et al., 2002), and prevention of DCM (Crone et al., 2002; Ozcelik et al., 2002). Her2/erbB2 has been a target for the development of therapeutic drugs. One major difficulty lies in the complexity of erbB2 signaling. It plays a role in multiple cell systems. Emerging evidence suggests that erbB2 is essential for transducing signaling elicited by multiple classes of ligands. Thus, it presents a great challenge to devise a selective treatment for a specific cell origin of disease. Comparative understanding of how erbB2 signals in cardiovascular and other cell systems will be important to the development of selective treatment of diseases involving deregulation of erbB2 function.

\section{A. THE erbB SIGNALING NETWORK}

NRGs play an important role in multiple cellular functions, including cell differentiation, migration, and survival. NRGs signal via ligand-induced dimerization between members of the epidermal growth factor receptor (EGFR) family of tyrosine kinase: namely, erbB2, erbB3, and erbB4. ErbB2 was identified as an oncogene due to its ability to induce the transformation of cultured fibroblasts (Yarden and Sliwkowski, 2001). ErbB2-overexpressing cancers usually are associated with a poor prognosis and account for about $30 \%$ of metastatic breast cancer in women.

Extensive biochemical data demonstrate that NRGs preferentially bind directly to erbB3 or erbB4 that, in turn, dimerize with other erbB receptors. The erbB2 receptor does not bind directly to any known ligands and functions primarily as a coreceptor for erbB3 and erbB4. Several biochemical studies have shown that erbB2 is the preferred dimerization partner for erbB3 and erbB4 upon NRG stimulation. Receptor dimerization results in auto- and trans-phosphorylation of intracellular domains. The phosphorylated forms of these receptors then can serve as docking sites for distinct cytoplasmic proteins involved in transducing downstream signaling cascades. The tyrosine residues that are phosphor-

ylated upon NRG binding determine the signaling molecules recruited for a particular signaling cascade (Yarden and Sliwkowski, 2001). 
Recently, two independent groups showed that the crystal structure of the erbB2 extracellular domain is a fixed, activated conformation (Cho et al., 2003; Garrett et al., 2003). Epidermal growth factor receptor (EGFR), erbB3, and erbB4 all undergo a ligand-dependent conformational change from an inactive to an active state. The active conformation displayed by erbB2 may help explain the potent signaling effects of this orphan receptor. Due to its active conformation, other signaling molecules can use erbB2 to rapidly transduce signals without the requirement of erbB2 ligand binding (Cho et al., 2003; Garrett et al., 2003). The timing, pattern, and tissue distribution of the erbB receptors, their ligand affinity, and their ability to homo- or heterodimerize underscore the diversity of the biological response resulting from distinct NRG-induced signaling pathways in target tissues.

A great deal of erbB2 signaling research has concentrated on cancer cells, with only limited studies on the signaling of NRGs in adult cardiac myocytes. The knowledge from other cell systems will provide insights into how erbB2 might signal in adult cardiac myocytes. ErbB2 has been shown to play a role in signaling elicited by interleukin (IL)- 6 in transfected prostate carcinoma cells, possibly through forming complexes with the gp130 subunit of the IL-6 receptor (Qiu et al., 1998). ErbB2 is transactivated by signaling of $G$ protein-coupled receptors (Daub et al., 1996). In human carcinoma cell lines, the integrin laminin receptors ( $\alpha 6 \beta 1$ and $\alpha 6 \beta 4$ ) associate with erbB2 (Falcioni et al., 1997). When these cells were treated with a monoclonal antibody to the $\alpha 6$ integrin, a ligand-dependent increase in erbB2 phosphorylation was seen. Cells expressing both erbB2 and $\alpha 6 \beta 4$ integrin showed enhanced proliferation rates and invasiveness, suggesting that this receptor interaction might contribute to a moremalignant phenotype in carcinoma cells. In a human breast cancer cell line, known as MCF-7 cells, HRG- $\beta 1$ results in Akt activation, which is blocked by antiestrogens. HRG- $\beta 1$, bound to the erbB2/erbB3 heterodimer, in the presence of membrane estrogen receptor (ER), interacts and activates phosphatidylinositol 3-kinase (PI3-K)/Akt. Akt leads to nuclear ER- $\alpha$ phosphorylation, thereby altering its expression and transcriptional activity. ErbB2 has been shown to interact with the $\mathrm{G}$ protein-coupled growth hormone $(\mathrm{GH})$ receptor in cancer cell lines (Huang et al., 2003). GH, by activating extracellular signal-regulated kinases (ERKs), can modulate epidermal growth factor (EGF)-induced EGF receptor (EGFR) trafficking and signaling, suggesting mechanisms of cross-talk between the $\mathrm{GH}$ and EGF/erbB2 signaling system.

In the heart, $G$ protein-coupled receptor agonists such as noradrenaline, endothelin-1, and angiotensin II are known to be involved in cardiac hypertrophy via transactivation of the EGFR (Shah and Catt, 2003). In addition, it has been shown that a serotonin (5-hydroxytryptamine, 5-HT) mouse model that expresses inactive 5HT-2B leads to embryonic and neonatal death and a specific reduction in erbB2 and lack of trabeculae in the heart. These in vivo data suggest that the 
Gq-coupled receptor 5-HT (2B) uses the signaling pathway of tyrosine kinase receptor erbB2 for cardiac differentiation (Nebigil et al., 2000). In cardiac myocytes, EGFR kinase activity is involved in protease-activated receptor (PAR)4-dependent (relative of cardiac myocyte G protein-coupled receptors) activation of the p-38 mitogen-activated protein kinase (MAPK) signaling pathway, again showing cross-talk between EGFR and G protein-coupled receptor signaling (Sabri et al., 2003).

Recently, it has been shown that heparin-binding EGF-like growth factor (HB-EGF)-null mice, when viable, develop severe heart failure with grossly enlarged ventricular chambers (Iwamoto et al., 2003). HB-EGF induces tyrosine phosphorylation of erbB2 and erbB4. The HB-EGF-null mice exhibited significantly lower levels of erbB2 and erbB4 phosphorylation, suggesting that HB-EGF is a cardiac survival factor partially through erbB2. Additional interactions between NRG and other signaling pathways in the heart, such as insulin-like growth factor-1 (IGF-1), have been described (Hertig et al., 1999). The extent to which the NRG signaling network participates in the signal transduction of other diverse signaling pathways in the adult heart needs further investigation. Whether erbB2 associates with other $\mathrm{G}$ protein-coupled receptors, integrins, or gp130 in adult cardiac myocytes is unclear. It is possible that the lack of trabeculae formation in the erbB2 mutant may be due to the inactivation of a number of signaling pathways in which erbB2 functions as a central mediator.

The identification of the NRG/erbB network as potential signal integrators in response to a variety of stimuli suggests that the loss of the primary coreceptor, erbB2, either by genetic deletion (transgenic mouse models) or Herceptin treatment, cripples the coordinated cross-talk between multiple signaling pathways, resulting in loss of cellular integrity and function.

\section{B. THE ROLE OF NRG SIGNALING IN CARDIAC DEVELOPMENT}

The most-extensively studied role of NRG-mediated signaling focused on its impact on the development and maintenance of the central and peripheral nervous systems (PNS). Knockout mice provide models with which to characterize the developmental and physiological role of a gene(s) of interest. Targeted deletion of the NRG1, erbB2, or erbB4 gene results in early embryonic lethality due to cardiac developmental defects (Gassmann et al., 1995; Lee et al., 1995; Meyer and Birchmeier, 1995). These mouse models provided the first in vivo evidence of the role of the NRG/erbB signaling network for proper cardiac development. It is important to note that neither erbB2 nor erbB4 alone could compensate for the loss of the other receptor in the heart, suggesting that NRG signaling in the heart requires erbB2/erbB4 heterodimers. The embryonic lethal erbB3-null mice display a 
cardiac phenotype distinct from the NRG and NRG receptor knockout mice. The loss of erbB3 expression in the cardiac cushion mesenchyme of erbB3null mice results in thin cardiac cushions and abnormal heart valve formation (Erickson et al., 1997; Camenisch et al., 2002).

\section{THE ROLE OF erbB2 IN DEVELOPMENT OF HYPERTROPHY AND CELL SURVIVAL}

Both erbB2 and erbB4 receptors are expressed in isolated neonatal and adult cardiac myocytes (Zhao et al., 1998). ErbB2/Her2 is localized to the transverse tubules (Ozcelik et al., 2002) and erbB4 is localized to the calveolar microdomains within cardiac myocytes (Zhao et al., 1999). In vitro, NRG promotes the survival of neonatal and adult cardiac myocytes and induces hypertrophic change, including changes in cell morphology, increased protein synthesis, and expression of embryonic genes (Zhao et al., 1998). A soluble neuregulin-1 (NRG1, recombinant human glial growth factor 2, rhGGF2) promotes the proliferation, survival, and growth of isolated neonatal and adult cardiac myocytes. RhGGF2 provoked a two-fold increase in embryonic cardiac myocyte proliferation. RhGGF2 also promotes survival and inhibited apoptosis of serumdeprived primary cultures and induces hypertrophic growth in both neonatal and adult ventricular myocytes, which is accompanied by an increase in expression of the hypertrophic marker prepro-atrial natriuretic factor (ANF) and skeletal $\alpha$-actin. NRG-1 was expressed in primary coronary microvascular endothelial cells prepared from adult rat ventricular muscle and its expression was increased by endothelin-1. This study suggested that the persistent expression of both NRG and its receptors in postnatal and adult heart poses a continuing role for the NRGs in the myocardial adaptation to physiological stress or injury. hrGGF increased protein synthesis and induced expression of ANF and sarcomeric F-actin polymerization in neonatal rat ventricular myocytes (Baliga et al., 1999). NRG-1 activated P42/44 MAPK extracellular signal-regulated kinase (ERK)-2/1 and ribosomal S6 kinase (RSK)-2, both of which could be inhibited by the ERK1antagonist PD-098059. The NRG receptors erbB2 and erbB4 are downregulated at both the message and protein levels in early stages of heart failure in animals with chronic hypertrophy secondary to aortic stenosis, suggesting a role for disabled erbB2 signaling in the transition from compensatory hypertrophy to failure (Rohrbach et al., 1999).

\section{D. erbB2 SIGNALING IN ANGIOGENESIS}

A study by Russell and colleagues (1999) suggests an important role for NRG in promoting angiogenesis via activation of the erbB receptors in endothelial cells. ErbB2, erbB3, and erbB4 are all highly expressed in endothelial cells. In human umbilical vein endothelial cell (HUVEC) culture experiments, NRG 
stimulation results in rapid calcium influxes and cell proliferation. In vivo, NRG administration led to the growth of new blood vessels in a rat corneal angiogenesis model. Antibodies directed against vascular endothelial growth factor (VEGF), in concentrations sufficient to block high levels of VEGF, had no effect on NRG-stimulated growth or tube formation of HUVEC cells. Therefore, NRG angiogenic effects appear to be independent of VEGF (Russell et al., 1999). A subsequent study showed that Herceptin acts as an anti-angiogenic factor when administered to Her2-overexpressing breast tumors in mice (Izumi et al., 2002). Herceptin also reduced the diameter and volume of tumor blood vessels, as compared to tumors treated with a control antibody. This expands our understanding of the antitumorigenic effects of Herceptin administration in the clinical setting.

\section{Experimental Models Investigating the Essential Role of erbB2}

\section{A. THE ROLE OF erbB2 IN NEURAL AND CARDIAC DEVELOPMENT}

We have investigated the developmental role of erbB2 by generating erbB2-null mice (Lee et al., 1995). In this study, the mutant embryos died at embryonic day (E) 11, probably as a result of dysfunctions associated with a lack of cardiac trabeculae. Development of cranial neural crest-derived sensory ganglia was markedly affected, as was development of motor nerves. Both erbB2 and NRGs are found in the neural crest cells and migrate out of the neural tube, suggesting that both molecules act via an autocrine mechanism to play an important role in the development and differentiation of neural crest cells. Trunk neural crest cells give rise to the neuronal cells of the dorsal root ganglion, whereas cranial sensory neurons are derived from cranial neural crest cells and placodal ectoderm (D'Amico-Martel and Noden, 1983; Le Douarin and Smith, 1988).

Whole-mount immunohistochemistry with TuJ1 antibodies against neuronal tubulin revealed that the mutant embryos had no immunoreactive staining in the dorsal portion of the trigeminal ganglion or the mandibular branch of the trigeminal ganglion. The dorsal-medial portion of the trigeminal ganglion was lost in the erbB2-null embryos. There were no axonal connections between the trigeminal ganglion and the hindbrain in sections through the whole ganglion. In mutant embryos, proximal portions of the glossopharyngeal and vagus nerves were considerably smaller than control embryos, suggesting that development of the superior and jugular ganglia was severely affected. Examination of the facial motor nerves suggested that neural crest-derived neurons in the facial ganglion also were affected. Considering the evidence of erbB2 expression in neural crest cells, the results of this study suggest that a lack of erbB2 affects the normal development of cranial neural crest-derived neurons but not placode-derived 
neurons at E10.5. As neural crest-derived trigeminal ganglion was markedly affected in erbB2-deficient embryos, we also investigated whether motor nerves were affected. Retrograde labeling showed that motor nerves fail to exit the hindbrain. It is likely that the development of trigeminal and facial motor nerves is secondary to a lack of the neural crest-derived portion of their respective sensory ganglia (Moody and Heaton, 1983).

\section{B. RESCUE OF THE CARDIAC TRABECULATION IN erbB2-NULL MICE}

Both erbB2 and NRGs are expressed in many non-neuronal tissues. In E9.5/10.5 embryos, erbB2 is detected in cardiac myocytes, whereas NRG is expressed in adjacent myocardium. This study suggested that erbB2 and NRG foster development of the heart via a paracrine mechanism. ErbB2 mice die of a trabeculation defect before gliogenesis in the PNS (Lawson and Biscoe, 1979) and before onset of neural muscular junction at E12 (Noakes et al., 1993; Piette et al., 1993). To study the role of erbB2 after E11, we genetically rescued the cardiac defect in erbB2-null mice by creating transgenic mice that expressed rat erbB2 under the control of a cardiac-specific $\alpha$-myosin heavy chain ( $\alpha$-MHC) promoter (Morris et al., 1999).

Mice expressing rat erbB2 in the heart were crossed with the erbB2-null mutants. The erbB2-null mutants that now overexpressed rat erbB2 in the heart formed cardiac trabeculae and survived to birth. However, due to the loss of innervations within the diaphragm muscle, the rescued mice were stillborn. Examination of the PNS in the rescued mutant embryos demonstrated a severe loss of both sensory and motor neurons and a complete absence of Schwann cell precursors in the peripheral nerves, demonstrating that erbB2 is required for normal development of the PNS (Morris et al., 1999). The prolonged viability of these mice provided further evidence supporting the important role of the NRG/erbB signaling network in the heart.

\section{CONDITIONAL HEART-RESTRICTED erbB2}

The NRG/erbB receptor signaling network has been established as a key modulator/regulator of multiple developmental and physiological processes, including cardiac development and adult cardiac function. Current technology allows for the generation of mice with both temporal and tissue-specific alterations in a gene(s) of interest, referred to as conditional mutants. The conditional knockout $(\mathrm{CKO})$ approach is useful to bypass early embryonic lethality, as observed in the NRG, erbB2, erbB3, and erbB4 total knockouts, as well as to define the cell-type, tissue-specific requirements of NRG signaling in the adult.

To investigate the role of erbB2 in the adult heart, we generated a line of erbB2 conditional mutant mice that lacked erbB2 in the heart and skeletal 
muscle. To generate the mice, we crossed our erbB2 floxed allele mouse line to a transgenic Cre line under control of the muscle creatine kinase (MCK) promoter, a Cre line shown to promote high-efficiency recombination in atrial and ventricular lineages and postnatal skeletal muscle. A second line of heartspecific erbB2-null mice was made by breeding erbB2 floxed mice with the myosin light chain $2 \mathrm{v}(\mathrm{MLC} 2 \mathrm{v})$ Cre transgenic line. The postnatal loss of erbB2 in the heart results in the progressive onset of DCM. The two independent, heart-restricted erbB2-null mouse lines display the same cardiac phenotype and differ only in the onset of DCM. Both these lines will be referred to as the erbB2 CKO mice.

The expression of erbB2 protein is significantly less in the adult hearts of erbB2 CKO mice, with no difference in the levels of the NRG receptor erbB4, compared to wild-type controls. The cardiomyopathy observed in the erbB2 CKO mice is consistent with that of dilated cardiomyopathy, including ventricular dilation, reactivation of embryonic gene expression, increase in heart:body weight ratio, and decreased cardiac contractility (Figure 1).

Transmission electron microscopy did not demonstrate alterations in cytoskeletal architecture but did reveal an increase in the number of mitochondria and vacuoles. The erbB2 CKO mutants have a significant increase in left ventricle (LV) end diastolic and end systolic dimensions (LVEDD and LVESD), decreased fractional shortening, decreased septal- and posterior-wall thickness, and decreased velocity of circumferential fiber shortening, compared to wild-type controls (Crone et al., 2002). In addition, retrograde catheterization of LV of the erbB2 CKO mutants revealed marked reduction in the maximum first derivative of $\mathrm{LV}$ pressure ( $\mathrm{LV} \mathrm{dP/dt} \max$ ), indicative of depressed myocardial contractility. No significant changes in heart rate, LV end diastolic pressure (EDP), and tau were observed between erbB2 $\mathrm{CKO}$ and controls. The decrease in $\beta$-adrenergic responsiveness in the erbB2 CKO mutants is consistent with that observed in human heart failure models. However, increasing doses of the $\beta$-adrenergic agonist, dobutamine, stimulates increased contractility and relaxation in the erbB2 CKO mice, segregating the erbB2 CKO mutants from other models of DCM. This suggests that erbB2 signaling is not required for this response.

Independently, Herceptin (Trastuzumab), a humanized monoclonal antibody directed against the extracellular domain of the Her2/erbB2 receptor, was used in clinical trials to treat female patients with metastatic breast cancer. Despite its potent ability to block erbB2-mediated signaling in breast, the cardiotoxic effects of Herceptin treatment were quite severe. This observation provided an in vivo link between suppression of erbB2 receptor signaling and impaired cardiac function and underscores the important role of the Her2/erbB2 receptor in adult cardiac function. Ozcelik and coworkers (2002) have independently reported similar results. Their study shows that erbB2 expression is localized to the transverse tubules of the cardiac myocytes. There was no increase in apoptosis 


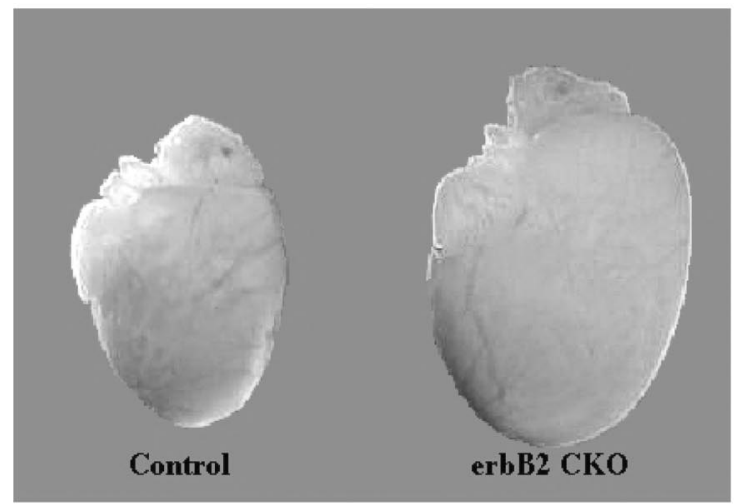

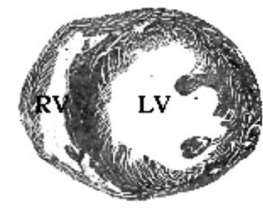

Control

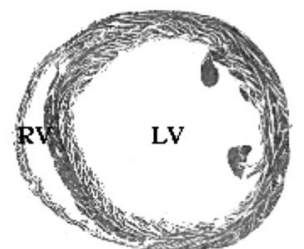

erbB2 CKO
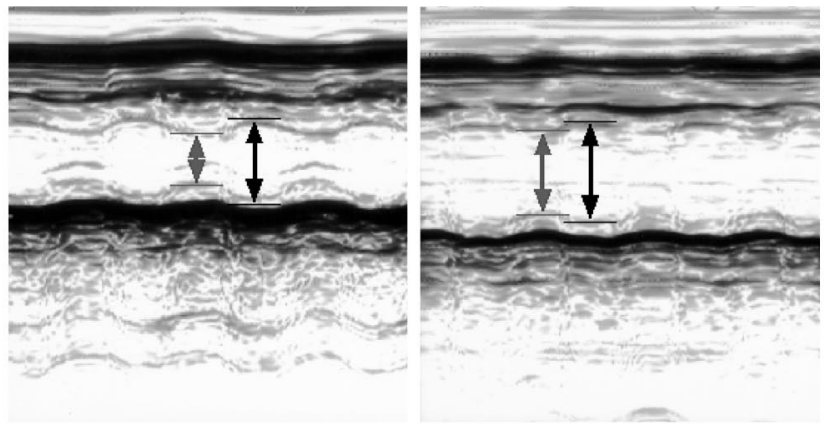

FIG. 1. ErbB2 conditional knockout (CKO) mice display multiple features of dilated cardiomyopathy. (Top panel) The heart from erbB2 CKO mutants is enlarged due to dilated left ventricle (LV), as shown in hematoxylin/eosin (H/E)-stained cross-sections of the heart (middle panel). Echocardiography (bottom panel) and hemodynamic measurements demonstrate an enlarged LV with thin walls and decreased contractility. Electron microscopy reveals intact cytostructural architecture but increased mitochondria and vacuolar structures (similar to features observed following anthracycline toxicity). ErbB2 CKO cardiomyocytes are more susceptible to anthracyline toxicity in culture. [Reprinted from Crone SA, Zhao YY, Fan L, Gu Y, Minamisawa S, Liu Y, Peterson KL, Chen J, Kahn R, Condorelli G, Ross J Jr, Chien KR, Lee KF 2002 ErbB2 is essential in the prevention of dilated cardiomyopathy. Nature Med 8(5):459-465, with permission of the Nature Publishing Group.] 
rates in erbB2 CKO mutants. The researchers concluded that erbB2 is not required for cardiac myocyte survival but is needed for adult cardiac function.

Although the majority of DCM is classified as idiopathic, it is now evident that there is a strong genetic component to the disease (Chien and Olson, 2002). In clinical trials for breast cancer therapy, Herceptin was an effective single agent for abolishing erbB2-mediated signaling. However, patients receiving Herceptin either following or concurrent with anthracycline treatment experienced an increased probability of developing DCM, from $7 \%$ to $28 \%$, respectively (Crone et al., 2002). Since the majority of cancer patients have received anthracycline treatment, the effects of Herceptin treatment alone have not been fully characterized. The erbB2 CKO mice are viable and display the same cardiac dysfunction as patients treated with Herceptin. To determine whether the erbB2 CKO cardiac myocytes are venerable to anthracycline-induced cell death, as shown in patients treated with Herceptin, we treated neonatal cardiac myocytes isolated from the erbB2 CKO mice with doses of Adriamyacin (anthracycline) ranging from 0.5 to 5 $\mathrm{mM}$. The erbB2 CKO mice displayed increased sensitivity to Adriamyacininduced cytotoxicity. It is clear that the cardiotoxic effects of Herceptin are a direct consequence of the loss of erbB2 signaling in the heart.

\section{Future Work}

The main focus of our laboratory's work is to understand the mechanism of how inactivation of erbB2 signaling in the adult heart results in DCM. We hope to identify the signaling pathways that are absent or inactive in the erbB2 CKO DCM mouse model. The erbB2 CKO mutants provide a model that will aid in the design of therapeutic drug targets to counteract the cardiotoxic effects of

Herceptin. Once the signaling pathways that are absent/altered in the erbB2 CKO mouse have been identified, Herceptin may be administered to all erbB2-positive cancers without resulting in cardiotoxicity.

\section{ACKNOWLEDGMENT}

Alejandra Negro and Bhawanjit K. Brar contributed equally to this work.

\section{REFERENCES}

Baliga RR, Pimental DR, Zhao YY, Simmons WW, Marchionni MA, Sawyer DB, Kelly RA 1999 NRG-1-induced cardiomyocyte hypertrophy. Role of PI-3-kinase, p70(S6K), and MEK-MAPK-RSK. Am J Physiol 277(5 Pt 2):H2026-H2037

Camenisch TD, Schroeder JA, Bradley J, Klewer SE, McDonald JA 2002 Heart-valve mesenchyme formation is dependent on hyaluronan-augmented activation of ErbB2-ErbB3 receptors. Nature Med 8(8):850-855 
Chein KR 1999 Stress pathways and heart failure. Cell 98(5):555-558

Chien KR, Olson EN 2002 Converging pathways and principles in heart development and disease. Cell 110(2):153-162

Cho HS, Mason K, Ramyar KX, Stanley AM, Gabelli SB, Denney DW Jr, Leahy DJ 2003 Structure of the extracellular region of HER2 alone and in complex with the Herceptin Fab. Nature 421(6924):756-760

Crone SA, Zhao YY, Fan L, Gu Y, Minamisawa S, Liu Y, Peterson KL, Chen J, Kahn R, Condorelli G, Ross J Jr, Chien KR, Lee KF 2002 ErbB2 is essential in the prevention of dilated cardiomyopathy. Nature Med 8(5):459-465

D'Amico-Martel A, Noden DM 1983 Contributions of placodal and neural crest cells to avian cranial peripheral ganglia. Am J Anat 166(4):445-468

Daub H, Weiss FU, Wallasch C, Ullrich A 1996 Role of transactivation of the EGF receptor in signalling by G-protein-coupled receptors. Nature 379(6565):557-560

Erickson SL, O'Shea KS, Ghaboosi N, Loverro L, Frantz G, Bauer M, Lu LH, Moore MW 1997 ErbB3 is required for normal cerebellar and cardiac development: a comparison with ErbB2-and heregulin-deficient mice. Development 124(24):4999-5011

Falcioni R, Antonini A, Nistico P, Di Stefano S, Crescenzi M, Natali PG, Sacchi A 1997 Alpha 6 beta 4 and alpha 6 beta 1 integrins associate with ErbB-2 in human carcinoma cell lines. Exp Cell Res 236(1):76-85

Garrett TP, McKern NM, Lou M, Elleman TC, Adams TE, Lovrecz GO, Kofler M, Jorissen RN, Nice EC, Burgess AW, Ward CW 2003 The crystal structure of a truncated ErbB2 ectodomain reveals an active conformation, poised to interact with other ErbB receptors. Mol Cell 11(2):495-505

Gassmann M, Casagranda F, Orioli D, Simon H, Lai C, Klein R, Lemke G 1995 Aberrant neural and cardiac development in mice lacking the ErbB4 neuregulin receptor. Nature 378(6555): 390-394

Hertig CM, Kubalak SW, Wang Y, Chien KR 1999 Synergistic roles of neuregulin-1 and insulin-like growth factor-I in activation of the phosphatidylinositol 3-kinase pathway and cardiac chamber morphogenesis. J Biol Chem 274(52):37362-37369

Huang Y, Kim SO, Jiang J, Frank SJ 2003 Growth hormone-induced phosphorylation of epidermal growth factor (EGF) receptor in 3T3-F442A cells. Modulation of EGF-induced trafficking and signaling. J Biol Chem 278(21):18902-18913

Iwamoto R, Yamazaki S, Asakura M, Takashima S, Hasuwa H, Miyado K, Adachi S, Kitakaze M, Hashimoto K, Raab G, Nanba D, Higashiyama S, Hori M, Klagsbrun M, Mekada E 2003 Heparin-binding EGF-like growth factor and ErbB signaling is essential for heart function. Proc Natl Acad Sci USA 100(6):3221-3226

Izumi Y, Xu L, di Tomaso E, Fukumura D, Jain RK 2002 Tumour biology: herceptin acts as an anti-angiogenic cocktail. Nature 416(6878):279-280

Lawson SN, Biscoe TJ 1979 Development of mouse dorsal root ganglia: an autoradiographic and quantitative study. J Neurocytol 8(3):265-274

Le Douarin NM, Smith J 1988 Development of the peripheral nervous system from the neural crest. Annu Rev Cell Biol 4:375-404

Lee KF, Simon H, Chen H, Bates B, Hung MC, Hauser C 1995 Requirement for neuregulin receptor erbB2 in neural and cardiac development. Nature 378(6555):394-398

Meyer D, Birchmeier C 1995 Multiple essential functions of neuregulin in development. Nature 378(6555):386-390

Michels VV, Driscoll DJ, Miller FA, Olson TM, Atkinson EJ, Olswold CL, Schaid DJ 2003 Progression of familial and non-familial dilated cardiomyopathy: long term follow up. Heart 89(7):757-761 
Moody SA, Heaton MB 1983 Developmental relationships between trigeminal ganglia and trigeminal motoneurons in chick embryos. Ganglion development is necessary for motoneuron migration. J Comp Neurol 213(3):327-343

Morris JK, Lin W, Hauser C, Marchuk Y, Getman D, Lee KF 1999 Rescue of the cardiac defect in ErbB2 mutant mice reveals essential roles of ErbB2 in peripheral nervous system development. Neuron 23(2):273-283

Nebigil CG, Choi DS, Dierich A, Hickel P, Le Meur M, Messaddeq N, Launay JM, Maroteaux L 2000 Serotonin 2B receptor is required for heart development. Proc Natl Acad Sci USA 97(17):9508-9513

Noakes PG, Phillips WD, Hanley TA, Sanes JR, Merlie JP 1993 43K protein and acetylcholine receptors colocalize during the initial stages of neuromuscular synapse formation in vivo. Dev Biol 155(1):275-280

Ozcelik C, Erdmann B, Pilz B, Wettschureck N, Britsch S, Hubner N, Chien KR, Birchmeier C, Garratt AN 2002 Conditional mutation of the ErbB2 (HER2) receptor in cardiomyocytes leads to dilated cardiomyopathy. Proc Natl Acad Sci USA 99(13):8880-8885

Piette J, Huchet M, Houzelstein D, Changeux JP 1993 Compartmentalized expression of the alphaand gamma-subunits of the acetylcholine receptor in recently fused myofibers. Dev Biol 157(1):205-213

Qiu Y, Ravi L, Kung HJ 1998 Requirement of ErbB2 for signalling by interleukin-6 in prostate carcinoma cells. Nature 393(6680):83-85

Rohrbach S, Yan X, Weinberg EO, Hasan F, Bartunek J, Marchionni MA, Lorell BH 1999 Neuregulin in cardiac hypertrophy in rats with aortic stenosis. Differential expression of erbB2 and erbB4 receptors. Circulation 100(4):407-412

Russell KS, Stern DF, Polverini PJ, Bender JR 1999 Neuregulin activation of ErbB receptors in vascular endothelium leads to angiogenesis. Am J Physiol 277(6, pt 2):H2205-H2211

Sabri A, Guo J, Elouardighi H, Darrow AL, Andrade-Gordon P, Steinberg SF 2003 Mechanisms of protease-activated receptor-4 actions in cardiomyocytes. Role of Src tyrosine kinase. J Biol Chem 278(13):11714-11720

Shah BH, Catt KJ 2003 A central role of EGF receptor transactivation in angiotensin II-induced cardiac hypertrophy. Trends Pharmacol Sci 24(5):239-244

Yarden Y, Sliwkowski MX 2001 Untangling the ErbB signalling network. Nat Rev Mol Cell Biol 2(2):127-137

Zhao YY, Sawyer DR, Baliga RR, Opel DJ, Han X, Marchionni MA, Kelly RA 1998 Neuregulins promote survival and growth of cardiac myocytes. Persistence of ErbB2 and ErbB4 expression in neonatal and adult ventricular myocytes. J Biol Chem 273(17):10261-10269

Zhao YY, Feron O, Dessy C, Han X, Marchionni MA, Kelly RA 1999 Neuregulin signaling in the heart. Dynamic targeting of erbB4 to caveolar microdomains in cardiac myocytes. Circ Res 84(12):1380-1387 